\title{
Valence Quark Polarization In the Nucleon And the
}

\author{
Deuteron Data
}

\author{
Firooz $\operatorname{Arash}^{(a) *}$ and Fatemeh Taghavi-Shahri ${ }^{(b)}$ \\ (a) Physics Department, Tafresh University, Tafresh, Iran \\ (b) Physics Department, Iran University of Science and Technology, Narmak, Iran
}

November 4, 2018

\begin{abstract}
Within the framework of the so-called valon model, We argue that a substantial part of the nucleon spin, about $40 \%$, is carried by the polarized valence quarks. The remaining is the result of cancelations between gluon polarization and the orbital angular momentum, where the gluon polarization is the dominant one. It is shown that the sea quark contributions to the spin of any hadron is simply marginal and consistent with zero. Our findings point to a substantially smaller value for $a_{8}$ than inferred from hyperon - $\beta$ decay, suggesting that full $S U(3)$ symmetric assumption needs to be reconsidered. New and emerging experimental data tend to support this finding. Finally, we show that within the model presented here the experimental data on the polarized structure functions $g_{1}^{p, n, d}$ are reproduced.
\end{abstract}

\section{INTRODUCTION}

The most direct tool and sensitive test for probing the quark and gluon substructure of hadrons is the polarized Deep Inelastic Scattering (DIS) processes. In such experiments

*e-mail: farash@cic.aut.ac.ir 
detailed information can be extracted on the shape and magnitude of the spin dependent parton distributions, $\delta q_{f}\left(x, Q^{2}\right)$. Deep inelastic scattering reveals that the nucleon is a rather complicated object consisting of an infinite number of quarks, anti-quarks, and gluon. Contributions from these components to the spin structure of hadron is an ongoing source of debates. Substantial activities, both in theory and in experiment, are performed to disentangle various contributions. The analysis of [1] reveals that the gluon and the orbital angular momentum contribute to the spin of nucleon with opposite signs. It turns out that the contribution from gluon polarization is the dominant factor, accounting for about $60 \%$ of the spin of proton. This still leaves a substantial amount of nucleon spin to come from quark sector: valence and sea. Expectations are that the sea is localized in the low $x$ region and the valence quark is dominant at $x \geq 0.3$. The role of sea quark is although still unclear, but both theoretical [1] and experimental investigations [2] [3][4] point in the direction that it should be marginal. The purpose of this letter is to investigate valence and sea quark contributions to the nucleon spin. COMPASS collaboration has recently published accurate data on the spin structure of deuteron [4] and has measured semiinclusive difference asymmetry, $A^{h^{+}-h^{-}}$, for hadrons of opposite charge in the kinematic region $0.006<x<0.7$. These measurements determine the valence polarization and allows for the evaluation of the first moment of $\Delta u_{v}+\Delta d_{v}$. COMPASS coll. have used a hybrid procedure, in that they have taken the MRST04 leading order parametrization of the unpolarized parton distributions along with the leading order fit of DNS polarized parton distributions [5] to evolve values of $\Delta u_{v}+\Delta d_{v}$ to a common $Q^{2}$ value fixed at $10(\mathrm{GeV} / \mathrm{c})^{2}$. The message of this experiment is threefold. First, they provide a set of accurate data on $g_{1}^{d}$, which is valuable in its own right. Secondly, they imply that the total sea quark contributions to the spin of nucleon is consistent with zero. This finding is in agreement with the earlier results from HERMES collaboration [2] [3] thus, reinforcing the point further. Neither group, however, provide an explanation for their findings. Thirdly, it seems that their results can accommodate both positive and negative gluon polarization, 
$\Delta G$.

It is important to investigate the implications of the new set of COMPASS data in an attempt to offer some explanations for these findings. The majority of the theoretical studies have been focused on the singlet component of the polarized structure function in order to explain its smallness. This has led to the assumption that the non-singlet component is rather well understood. Experimental evidence for this assumption comes from the confirmation of the Bjorken sum rule which relates the first moments of $g_{1}^{p}$ and $g_{1}^{n}$. However, the Bjorken sum rule depends merely on the fundamental $S U(2)$ isospin symmetry between the matrix elements of charged and neutral axial currents and therefore expected to hold, does not entirely fix the first moment of non-singlet component of $g_{1}^{p}$. This component, in the leading order, depends on $a_{8}$ which is under the assumption of $S U(3)_{f}$ symmetry usually taken to be $3 F-D=0.579 \pm 0.025$. Information that can be obtained from baryonic $\beta$ decay is highly limited and only provides information about the first moment of non-singlet part of $g_{1}^{p}$. As a result, when it is used to extract sea quark contributions to the proton spin, the outcome becomes less reliable. In the model described in the next section, we will show that the calculations in the framework of NLO perturbation theory, shows that the sea quark contribution to the spin of proton is essentially consistent with zero and the value for $a_{8}$ is substantially different from $0.579 \pm$ 0.025 , a result that seems confirmed by the emerging experiments.

\section{The Model}

As in [1], our approach in addressing the above issues is based on the so-called valon model. In this model a nucleon is composed of three dressed valence quarks, the valons. Each valon has its own internal structure which can be probed at high enough $Q^{2}$. At low $Q^{2}$, a valon behaves as a valence quark. The internal structure of a valon is calculated in the Next-to-Leading Order in QCD. We have worked in $\overline{M S}$ scheme with $\Lambda_{Q C D}=0.22$ 


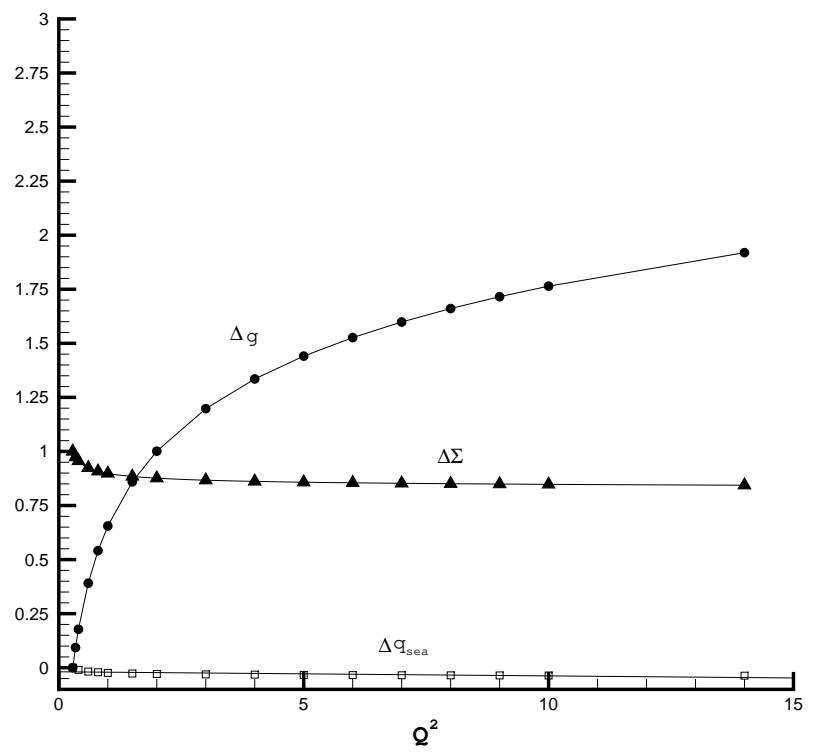

Figure 1: First Moments, $\Delta g\left(n=1, Q^{2}\right), \Delta \Sigma\left(n=1, Q^{2}\right)$, and $\Delta q_{\text {sea }}\left(n=1, Q^{2}\right)$ of various components in a valon as a function of $Q^{2}$

$\mathrm{GeV}$ and $Q_{0}^{2}=0.283 \mathrm{GeV}^{2}$. The details are given in [1]. It turns out that in a polarized valon the sea quark polarization is consistent with zero, whereas the valence quark carries almost entire spin of the valon. In fact, as it is evident from Figure 1, for $Q^{2} \geq 1$ $G e V^{2}$ the value of $\delta \Sigma_{\text {valon }} \simeq 0.88$ and remains so, almost independent of $Q^{2}$. Between $Q_{0}^{2}=0.283 \mathrm{GeV}^{2}$ and $\sim 1 \mathrm{GeV}^{2}$ it decreases from unity to 0.88. For the same range the value of $\delta q_{\text {sea }}$ for individual flavor is around -0.002 and hence, marginal. The fact that sea quark polarization in the valon is consistent with zero can be understood on the theoretical grounds. The valon structure is generated by perturbative dressing in QCD. In such processes with massless quarks, helicity is conserved and therefore, the hard gluons cannot induce sea polarization perturbatively. So, it appears that $\delta \Sigma_{\text {valon }}$ is very close to unity. However, the gluon polarization in a valon is positive and increases with $Q^{2}$, making the spin picture of a valon rather bluer. Spin of a valon is $\frac{1}{2}$ and the following 


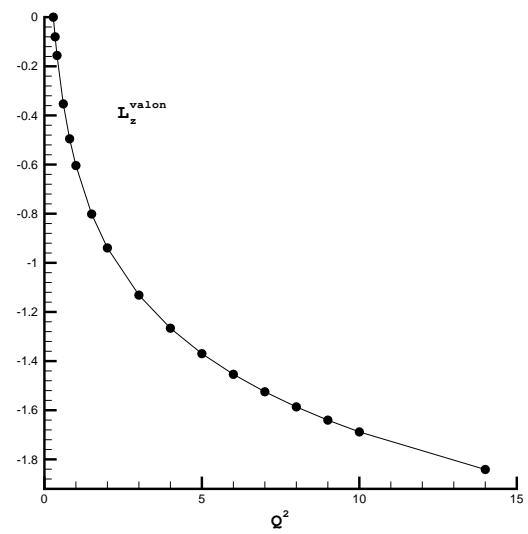

Figure 2: Total orbital angular momentum of partons, $L_{z}^{\text {valon }}\left(Q^{2}\right)$, in a valon as a function of $Q^{2}$.

sum rule applies.

$$
\frac{1}{2}=\frac{1}{2} \Delta \Sigma+\Delta g+L_{z}
$$

This picture shows that the gluon polarization in a valon is almost entirely compensated by the negative orbital angular momentum contribution. We would like to make it clear that in this work we have not attempted to solve evolution equation for $L_{z}$; it is determined simply by evaluating other quantities in Eq.(1) and then solving it for $L_{z}$. In Figure 2, we present the total orbital angular momentum of the partons in a valon. P.G. Ratcliffe [6] was the first to argue that the orbital angular momentum has a negative contribution to the spin of proton. In a remarkable paper by Ji, Tang, and hoodbhoy [7] it is shown that in the nucleon both the orbital angular momentum and the helicity of gluon grow with opposite signs. It is also shown that the total orbital angular momentum carried by quark and gluon is negative; a conclusion that we have also arrived at. In a recent paper [8] Anthony Thomas has also concluded that based on the standard features of the non-perturbative structure of the nucleon, the majority of the proton spin comes from the orbital angular momentum of $u$ and $\bar{u}$ quarks. Having specified the various components 
that contribute to the spin of a valon, we now turn to the hadron structure, which is obtained by a convolution integral as follows

$$
g_{1}^{h}\left(x, Q^{2}\right)=\sum_{\text {valon }} \int_{x}^{1} \frac{d y}{y} \delta G_{\text {valon }}^{h}(y) g_{1}^{\text {valon }}\left(\frac{x}{y}, Q^{2}\right)
$$

where $\delta G_{\text {valon }}^{h}(y)$ is the helicity of the valon in the hosting hadron and $g_{1}^{\text {valon }}\left(\frac{x}{y}, Q^{2}\right)$ is the polarized structure function of the valon. The latter, say, for a U-type valon, is simply given by

$$
2 g_{1}^{U}\left(z, Q^{2}\right)=\frac{4}{9}\left(\delta G_{\frac{u}{U}}+\delta G_{\frac{\bar{u}}{U}}\right)+\frac{1}{9}\left(\delta G_{\frac{d}{U}}+\delta G_{\frac{\bar{d}}{U}}+\delta G_{\frac{s}{U}}+\delta G_{\frac{\bar{s}}{U}}\right)+\ldots
$$

$\delta G_{\text {valon }}^{h}(y)$, is obtained from unpolarized valon distribution by

$$
\delta G_{j}(y)=\delta F_{j}(y) G_{j}(y)
$$

where, $G_{j}(y)$, are the unpolarized valon distributions and are determined by a phenomenological argument for a number of hadrons[9] [10] [11]. The functions $\delta F_{j}(y)$, where $j$ refers to $\mathrm{U}$ and $\mathrm{D}$ type valons, are given in [1]. In Figure 3 the shape of $\delta G_{U, D}(y)$ are shown. The helicity distributions of various parton types in a hadron is obtained by

$$
\delta q_{\frac{i}{h}}\left(x, Q^{2}\right)=\sum \int_{x}^{1} \frac{d y}{y} \delta G_{\frac{v a l o n}{h}}(y) \delta q_{\frac{i}{\text { valon }}}\left(\frac{x}{y}, Q^{2}\right)
$$

Here It suffice to give the parametric form of these helicity distribution functions in the proton

$$
x \delta q_{\frac{i}{p}}\left(x, Q^{2}\right)=a_{i} x^{b_{i}}(1-x)^{c_{i}}\left(1+d_{i} x^{0.5}+e_{i} x+f_{i} x^{2}\right)
$$

where $a_{i}, b_{i}, \ldots$ are functions of $Q^{2}$ and the index $i$ refers to $u_{\text {valence }}, d_{\text {valence }}$, sea quark, and gluon.

\section{Comparison with the experimental data}

We are now in a position to consider the experimental data at the hadron level. Substituting Eqs. (3) and (4) into equation (2) gives the polarized structure of the proton and 


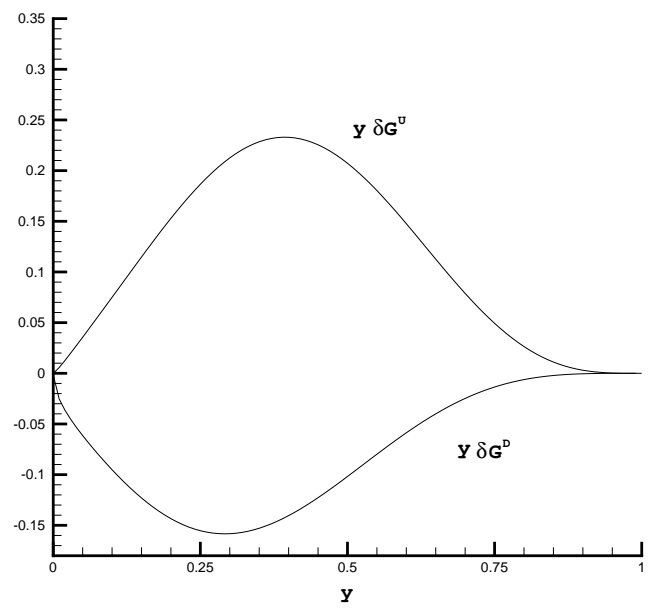

Figure 3: Polarized valon distributions, $y \Delta G_{U, D}(y)$ in proton

the neutron. The polarized structure function of deuteron is obtained by evaluating

$$
g_{1}^{d}=\frac{1}{2}\left(g_{1}^{p}+g_{1}^{n}\right)\left(1-1.5 \omega_{D}\right)
$$

We have calculated $g_{1}^{d}$ at $Q^{2}=10(\mathrm{Gev} / \mathrm{c})^{2}$ and $Q^{2}=3(\mathrm{Gev} / \mathrm{c})^{2}$ and compared our results with the experimental data from [4] along with the other global fit results in Figure 4. In contrast to DNS, AAC, and BB fits, our results are obtained without any fit to the experimental data. As stated earlier, the sea polarization in a valon is very small and hence, more so at the hadron level, being consistent with zero. This finding is interesting and is in excellent agreement with earlier results from HERMES collaboration [2] [3]. COMPASS collaboration although does not provide direct information about the sea polarization, but clearly indicates that their data is consistent with $\Delta \bar{u}_{\text {sea }}+\Delta \bar{d}_{\text {sea }} \sim 0$ and suggests that $\Delta \bar{u}_{\text {sea }}$ and $\Delta \bar{d}_{\text {sea }}$, if different from zero, must be of opposite sign [12]. Forthcoming COMPASS data on proton target will provide separate determinations of $\Delta \bar{u}_{\text {sea }}$ and $\Delta \bar{d}_{\text {sea }}$. The reconfirmation of HERMES findings on sea polarization as well as our results, naturally shifts the attention to the valence and gluon polarizations. The situation with the strange sea quark polarization is less clear because, the information 

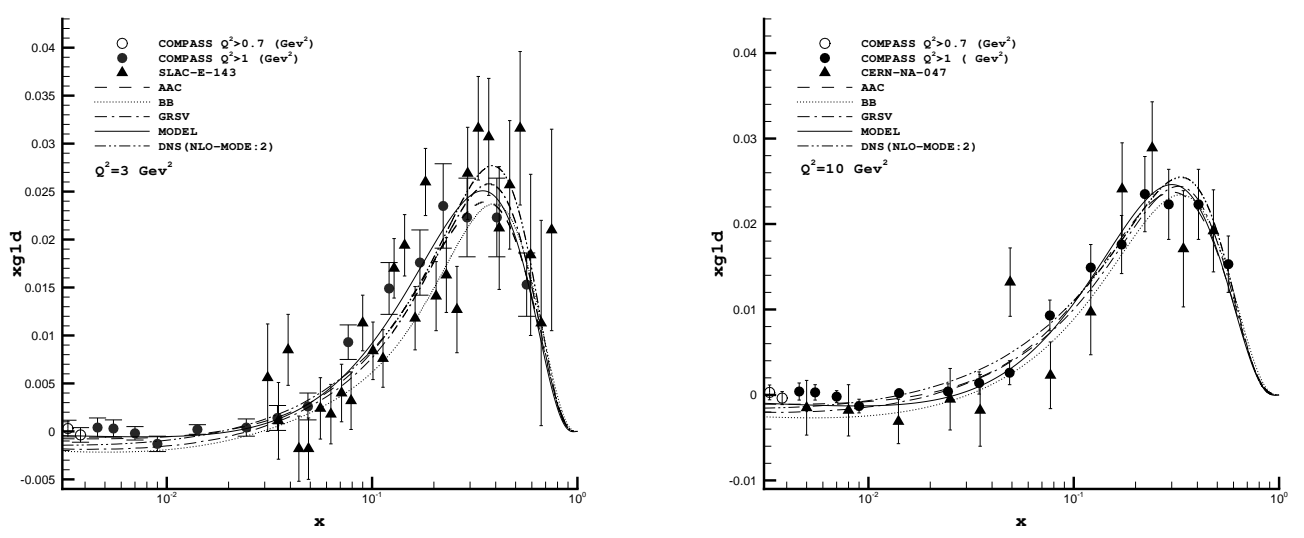

Figure 4: Deuteron structure function $g_{1}^{d}$ at $Q^{2}=3$ and $Q^{2}=10 \mathrm{GeV}^{2}$. For the purpose of comparison results from global fits are also shown.

about $\Delta s$ and $\Delta \bar{s}$ is obtained mainly from hyperon $\beta$ decay via the value of $a_{8}=3 F-D=$ $0.579 \pm 0.025$ or in models like the Nambu-Jona-Lasinio models. The way that $a_{8}$ is determined is based on the full $S U(3)$ symmetry between hyperon decay matrix elements of the flavor-changing weak axial and neutral currents. However, there are serious objections to this approach [13][14][15]. The results obtained from Nambu-Jona-Lasinio model also is not free from ambiguities [16]. Our findings for $a_{8}$ is 0.39 . This value is substantially smaller that the value inferred from hyperon data, but there are already experimental evidences in support of it. HERMES collaboration [17] gives the value $0.274 \pm 0.026 \pm 0.011$ in the measured range of $0.02<x<0.6$. The newer and more accurate data from COMPASS coll. which is extended the measured range to $0.006<x<0.7$ disfavors the assumption of a flavor symmetric polarized sea at a two $\sigma$ level and sets the value of $a_{8}$ at $0.40 \pm 0.07 \pm 0.05$. A detailed analysis of non-singlet structure functions in the nextto-leading order is given in [18] where the assumption of $S U(3)$ symmetry is studied and interesting conclusions about $a_{8}$ and $a_{3}$ are arrived. In contrast to $a_{8}$, our results in [1] for the Bjorken sum rule, or equivalently, $a_{3}$, is rather accurate and agrees with the accepted value $a_{3}=F+D=g_{A}=1.2573 \pm 0.0028$. 
Accepting HERMES results [17][19] and the data from COMPASS collaboration [4,12] points to the fact that the role of sea polarization is marginal and consistent with zero. This fact is naturally explained in our model, which relies on the Next-to-Leading order calculations in perturbative QCD. The HERMES Collaboration has just published [20] a new set of data, based on the charged-kaon production in deep inelastic scattering on the deuteron. Their new findings and analysis is essentially a reconfirmation of our results; that is, strange sea quark helicity distribution is zero within the experimental uncertainties [20]. Furthermore, they find that $a_{8}=0.285 \pm 0.046 \pm 0.057$. This situation leaves us with the valence and gluon polarization and the orbital angular momentum in the nucleon. The gluon and orbital angular momentum contributions to the spin of proton is discussed in [1]. Here our focus is on the valence sector. Our findings are shown in Figures 5 at $Q^{2}=10 \mathrm{GeV}^{2}$. Our results for $\Delta \Sigma$, the total quark contribution to the spin of proton, with $\Delta q_{\text {sea }} \sim 0$, lies in the range of $0.410-0.420$ for $Q^{2}=[1,10] \mathrm{GeV}^{2}$. The variation of $\Delta \Sigma$ is due to (marginal) $Q^{2}$ dependence of $\Delta q_{v}$ in the Next-to-Leading Order; since $\Delta P_{N S}^{(1)} \neq 0$. This value of $\Delta \Sigma$ is in excellent agreement with the results from COMPASS experiments [12] which gives

$$
\Gamma_{v}=\int_{0}^{1} d x\left(\Delta u_{v}(x)+\Delta d_{v}(x)\right)=0.41 \pm 0.07 \pm 0.05
$$

and with the results of HERMES [20] which is $0.359 \pm 0.026 \pm 0.018$.

\section{Conclusions}

We have shown that the valon representation is a suitable phenomenological frame work to deal with the polarization structure functions of hadrons. Within this representation a valon is a dressed quark and its spin arises from an interplay among the spin of the valence quark, the gluon polarization and the orbital angular momentum components. It also shows that the sea quark polarization in a valon is marginal. A negative orbital angular momentum emerges and competes with the gluon polarization, resulting in the 

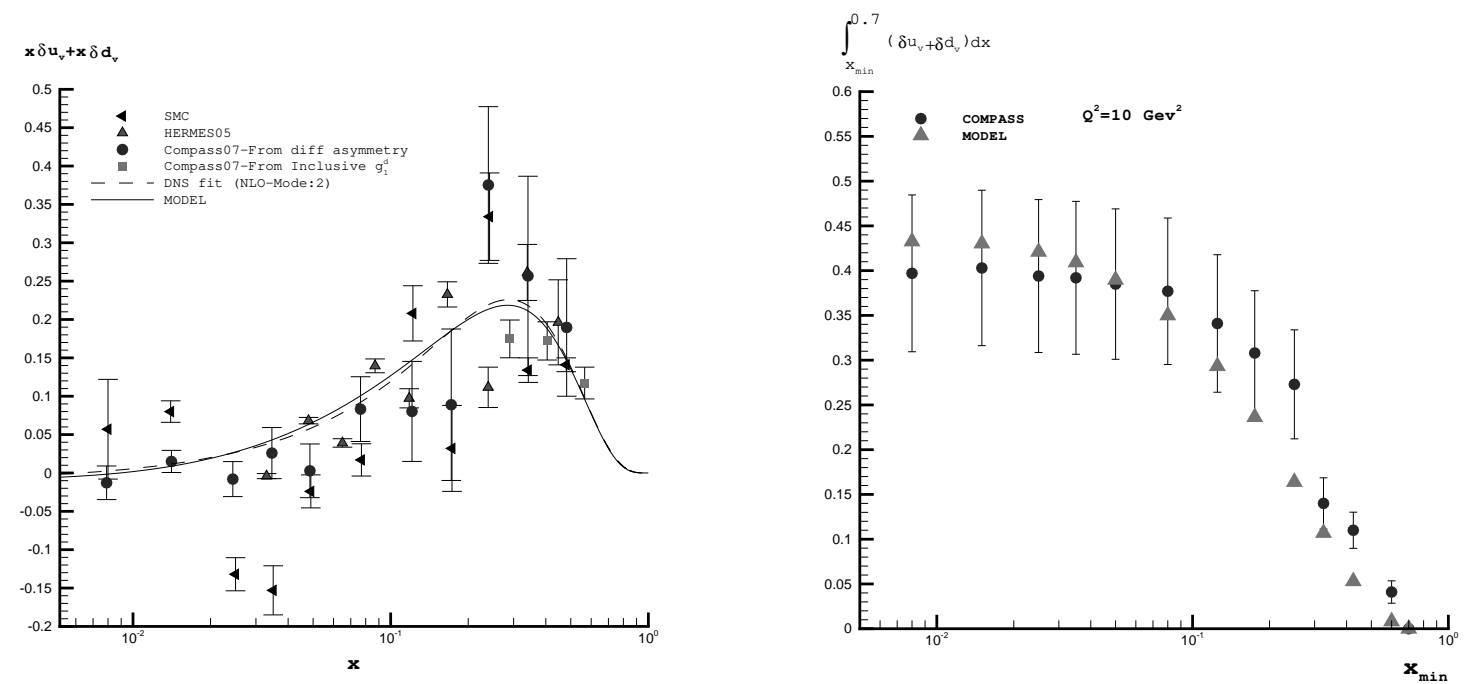

Figure 5: Left: $x \delta u_{v}(x)+x \delta d_{v}(x)$ at $Q^{2}=10\left(\frac{\mathrm{GeV}}{\mathrm{c}}\right)^{2}$.The data points are from [?] [2] [3] [10]. Full line curve is the model results and the dashed curve in DNS next-to-leading order fit results. Right: The integral of $\Delta u_{v}(x)+\Delta d_{v}(x)$ over the range $0.006<x<0.7$ measured by COMPASS coll. as the function of the low $x$ limit of integration, $x_{\text {min }}$ evaluated at $Q^{2}=10\left(\frac{\mathrm{GeV}}{\mathrm{c}}\right)^{2}$. The results from the model calculation are compared with the experimental data.

partial cancelation of the later. There is no net sea polarization at the hadron level; a result that agrees rather well with the existing experimental data. The valence quark contribution to the spin of proton stands at around $40 \%$ and the remaining comes from the gluon polarization. We have further shown that the results of this model predicts a substantially smaller value for $a_{8}$ than inferred from the hyperon $\beta$ decay. Experimental evidences for this finding is also gradually emerging, suggesting that the assumption of full $S U(3)$ symmetric sea needs reassessments. Finally, we have demonstrated that the model accommodates the wealth of experimental data on the polarized structure functions, $g_{1}^{p}, g_{1}^{n}, g_{1}^{d}$ rather nicely.

\section{References}

[1] Firooz Arash and Fatemeh Taghavi-Shahri, JHEP 07 (2007)071. 
[2] A. Airapetian, et al. (HERMES Coll.), Phys. Rev. Lett. 92:012005, 2004.

[3] A. Airapetian, et al. (HERMES Coll.), Phys. Rev. D 71: 012003, 2005.

[4] COMPASS Collab. V. Yu. Alexakhin et al., Phys. Lett. B 647 (2007) 8.

[5] D. de Florian, G. A. Navarro, R. Sasso, Phys. Rev. D 71 (2005) 094018.

[6] P.G. Ratcliffe, Phys. Lett. B 192180.

[7] Xiangdong Ji, Jian Tang, and Pervez hoodbhoy, Phys. Rev. Lett. 76, 740 (1996).

[8] Anthony W. Thomas, arXive:0803.2775

[9] F. Arash, A. N. Khorramian, Phys. Rev. C 67 (2003) 045201.

[10] F.Arash, Phys. Lett. B 557 (2003) 38.

[11] F. Arash, Phys. Rev. D 679 (2004) 054024.

[12] COMPASS Collab. V. Yu. Alexakhin et al. archiv 0707.4977

[13] M. Anselmino, B. L. Ioffe, and E. Leader, Sov. J. Nucl. Phys. 49 (1989) 136.

[14] D. B. kaplan, A. Manohar, Nucl. Phys. B 310 (1988) 527.

[15] H. J. Lipkin, Phys. Lett. B 256, 284 (1991); 337, 157 (1994); J. Lichtenstadt and H.J. Lipkins, ibid. 353, 119 (1995).

[16] K. Steininger and W. Weise, Phys. Lett. B 329, 169 (1994).

[17] H. E. Jackson, AIP Conf. Proc. 842 (2006) 363; hep-ex/0601006.

[18] M. Stratman, A. Weber, and W. Vogelsang, Phys. Rev. D 53 (1996) 138.

[19] HERMES Coll., A. Airapetian, et al., Phys. Rev. D 75: 012007, 2007.

[20] A. Airapetian, et al. (HERMES Coll.), hep-ex/ 0803.2993 v1. 